\title{
A Framework for Research and Practice: Relationship between Expatriate Knowledge Transfer, Subsidiary Absorptive Capacity, and Subsidiary Performance
}

\author{
Rui Yang, Xiaowen He \\ South China University of Technology, Guangzhou, China \\ Email: andrew.yang_scut@foxmail.com
}

Received November 23, 2013; revised December 26, 2013; accepted January 11, 2014

Copyright (c) 2014 Rui Yang, Xiaowen He. This is an open access article distributed under the Creative Commons Attribution License, which permits unrestricted use, distribution, and reproduction in any medium, provided the original work is properly cited. In accordance of the Creative Commons Attribution License all Copyrights @ 2014 are reserved for SCIRP and the owner of the intellectual property Rui Yang, Xiaowen He. All Copyright (C) 2014 are guarded by law and by SCIRP as a guardian.

\begin{abstract}
It plays an important role in establishing and maintaining competitive advantages in multinational corporations (MNCs). MNCs transfer knowledge among parent corporations and foreign subsidiaries. While multinational firms tend to send outstanding expatriates to foreign subsidiaries to transfer knowledge. In this study, we theoretically indentify three dimensions during the knowledge transfer among expatriates and local staff: ability, motivation and seeking for the opportunities to transfer knowledge. Then we integrate the ability-motivation-opportunity with the absorptive capacity of corporations. The result shows that knowledge transfer from expatriates has indirect impact on performances of foreign subsidiaries, which will be stronger as the absorptive capacity of corporation becomes stronger.
\end{abstract}

\section{KEYWORDS}

Knowledge Transfer; Multinational Corporations; Expatriates

\section{Introduction}

As technology develops and global integration accelerates, the core competitive advantages of corporations change from resource and wealth to knowledge management and innovation. Tencent in 2012 was named one of the world's top ten most innovative companies. The CEO of Tencent, Huateng Ma said, business and finance are not the most important things for Tencent. Business can be expanded and replaced. Finance can be absorbed and adjusted, while human resource can not be replaced easily and it's our most valuable asset. The essence of human resource is that human can offer knowledge for corporations, and knowledge is the basic of competitive advantage, which has been agreed by many scholars.

Competitive advantages of MNCs have increasingly depended on their unique knowledge which accumulates during their long-term development and effective knowledge transfer between parents and foreign subsidiaries. MNCs need to integrate international human resource, in order to make full use of human resource of foreign subsidiaries. While the integration of these knowledge to some extend depends on expatriates sent from parent company to foreign subsidiaries. Lee thinks about that successful expatriates staff dispatch can not only increase international reputation and profits of MNCs, but also can expand international market. In the previous study, scholars hope to find out relationship between expatriates staff and the performance of subsidiaries, which the results aren't consistent with. For example, in the research of Gong, he pointed out that expatriates staff is positively related to the performance of subsidiaries, while Gaur in 2007 found out that the relationship between expatriates staff and performance of subsidiaries is not so significant or even some evidences showed negative correlation. However, most of these researches focus on the number and the characteristics of expatriates staff, and there is a big uncertainty during the research. Based on this, we change our focus on theoretically indentifying three dimensions during the knowledge transfer among expa- 
triates: ability, motivation and seeking for the opportunities to transfer knowledge. Then we come up with a model of knowledge transfer, the absorptive capacity of subsidiaries and the performance of subsidiaries to do the study.

\section{Method}

In order to achieve the objection of the research, we mainly use literature research method to do the study. Literature research mainly refers to do the research by collecting identifying and reorganizing exiting literature, and then come up with a scientific cognition of the research topic. There are two aspect of the value of the literature research. One is that we can get new argument, new research perspective and new idea through doing the literature research. The other is that we can get revelation and evidence for our view from the previous researches. In our study, we use literature research method to collect relative previous research results and do the analysis basis on the previous research, then come up with the idea that ability, motivation and seeking for the opportunities to transfer knowledge are the key elements through the knowledge transfer among expatriates and local staff. What's more, we get evidence to analyze the relationships among the ability-motivation-opportunity, the absorptive capacity of corporations and the performance of subsidiaries.

\section{Model and Hypothesis of Knowledge Transfer from Expatriates}

As the development of economic globalization, multinational companies emerge. Until 2011, there are 70,000 MNCs around the world. As there are more and more subsidiaries around the world, staff turnover increases, especially knowledge staff delivers excellent technology and management knowledge from parent companies to subsidiaries, which leads to expatriates. The research from Anders Edstrom and Jay R. Galbraith shows that there are three mainly reasons to explain why MNCs send expatriates to subsidiaries, which are full fill their vacancies, cultivate human resource and promote organizational development [1]. As we text input "expatriates" in Cnki.net as the key word to search, we can find out that there are 155 papers about "expatriates” from 1949 until now. However, only one of these 155 papers is about knowledge transfer. As globalization is developing in China, Chinese corporations need some study to solve the problems of staff turnover. From the 155 papers, we can find out that most of the papers focus on cross-cultural adaptation among the expatriates, the salary and welfare system and technology transfer of expatriates. Though some scholars had done some research about knowledge transfer, these researches were just from the aspect of cross-cultural communication among expatriates, and there are no research about the ability to transfer knowledge and the willingness to seek knowledge of expatriates.

The concept of knowledge transfer was first proposed by Teece in 1997. Knowledge transfer can be identified as the process that people positive to transfer knowledge, and knowledge transferred by the people in different organization in different ways. In the following study, scholars found out that the role of knowledge transfer in MNCs especially significant. Gupta and Govindarajan found out that we could conclude resource flow inside the MNCs into finance flow, product flow and knowledge flow, among which knowledge flow is the most important. At the same time, they came up with the idea that knowledge flow to five direct: 1 ) from parent to subsidiaries, 2) from subsidiaries to parent, 3) from the host country to subsidiaries, 4) from subsidiaries country to the host, 5) from subsidiaries to subsidiaries [4]. While parent companies send expatriates to subsidiaries, it aims to transfer knowledge from parent companies to subsidiaries, as the result that subsidiaries can agree with the culture of the parent companies and make profits. Basic on the precious studies, many scholars keep an eye on the knowledge and technology skill of expatriates, because they thought the knowledge and technology skill are the basic of the knowledge transfer. On the other side, Arvey found out that the aim to transfer plays a very important role [2]. At last, Chang found out that expatriates seek the chances to transfer knowledge also is very important to impact the effect of knowledge transfer [3]. In our study, we integrate ability, motivation and seeking chances to transfer as the competitive advantages of expatriates.

The ability to transfer: Knowledge is always lurking inside the employee initially. Individual knowledge is the foundation of organizational knowledge [3]. The ability of knowledge of expatriates can mainly divide into two aspects, which are management skills and expertise skills. In 2002, Antal did a long-term research in two German companies, and after the research, he found that the knowledge which expatriates transfer to subsidiaries can be divided into five kinds, which are explicit knowledge, tacit knowledge, contextual knowledge, self-gain knowledge and relationship knowledge. Then Gerhard Fink came up with an idea that knowledge that expatriates transfer includes marketing knowledge, personal skills, management skill and network skill. Basic on the precious research, we can divide expertise skills into 1) products, packaging and process design knowledge and skill, 2) production, processing and manufacturing skill, 3) procuring, warehousing and logistics skill. Management skills includes: 1) operation, brand maintenance and sale skill, 2) human resource management skill 3) corporation cultural, strategy and value management skill. Szulanski 
come up with an idea that the ability to transfer can divide into three aspects, which include the ability to represent knowledge, assess the ability of the recipient, transform the knowledge to use in new environment. If the ability to transfer knowledge of expatriates is strong, the staff of subsidiaries will get more advance knowledge from the expatriates, which results that subsidiaries can transform the knowledge into productive forces and improve the performance of subsidiaries. We make the following hypothesis:

H1-1: While the ability of knowledge of expatriates is stronger, the performance of subsidiaries will be better.

The motivation to transfer: Expatriation is an effective tool to collect and maintain knowledge base. It plays an important role for knowledge transfer whether expatriates are willing to deal with some cultural barriers and not afraid of knowledge stolen. Basic on previous researches, we can divide motivation into intrinsic motivation and extrinsic motivation. Some scholars also did some research on how extrinsic rewards impact intrinsic motivation. However, most of these researches focus on enterprise, and few research is about boundary-spanning [4]. Intrinsic motivation is important to transfer knowledge between parent enterprises and subsidiaries, especially for creative job and mutil-task work. Mutil-task work is work whose object can be clearly identified. Basic on this, we can know intrinsic motivation is important to transfer knowledge [5,6]. We can measure motivation to transfer knowledge from five aspects: 1) whether expatriates are willing to solve cultural barriers to transfer knowledge; 2) whether expatriates teach staff in subsidiaries without reservation; 3) whether expatriates are willing to overcome technical and other difficulties to help subsidiaries; 4) whether expatriates are willing to invest time and energy to teach advantage skills; 5) whether expatriates can insist to transfer culture and technical skill. As we all know, there are difficulties during the knowledge transfer among expatriates and local staff. If expatriates are willing to transfer knowledge, they can overcome all the difficulties to transfer knowledge to subsidiaries, which will actively improve the performance of subsidiary. Then we put forward the following hypothesis:

H1-2: While the motivation of expatriates to transfer knowledge is stronger, the performance of subsidiary is better.

Seeking opportunities to transfer: MNCs encourage staff actively interacting with expatriates [7]. Many expatriates are willing to talk with staff in subsidiaries and to teach knowledge and skill. The essence of seeking opportunities to transfer is to seek a transfer channel. Ghoshal and Bartlett found out that if there is no transfer channel, knowledge transfer can not happen. Until now, many scholars came up with the idea that builds formal and informal integration mechanism to transfer knowledge. Formal integration mechanism is a coordination model basic on formal systems, police and standards. Both sides transfer knowledge though constraints and conditions, which we name it as knowledge communication under system. Inkepen put forward that if parent companies transfer knowledge to subsidiaries, parent companies must build a core knowledge transfer system [8]. Socialization mechanisms are established on a system that different companies transfer knowledge under individual intimate relationship. This system is similar to informal communication, which aim is to build common beliefs, objections and value inside companies (Nohria \& Ghoshal, 1994). At the same time, people make more connections during transferring knowledge. We call this kind of communication as socialization communication. Some scholars have done some research on seeking opportunity to transfer. Jiabin Yi (2012) found out that members have different ideas in age, race, value and so on, which result as structural conflict, stock-based conflict and relationship conflict. These conflicts will form barriers on the process to transfer knowledge. Knowledge conflicts will form an invisible boundary. Gang Zhang concluded that there are three forms of knowledge conflict, which are the conflict between explicit and tacit knowledge, expert knowledge conflict and the conflict between existing knowledge and new knowledge in team [8]. In the process of knowledge transfer in MNCs, the main differences between parent companies and subsidiaries are technology, geographic, organizational and cultural differences [9]. Only when these four differences become a psychological contract fulfillment, the opportunity to transfer knowledge rises. At the same time, Chao Wang said the key point to catch the opportunity is to understand the implied knowledge. In a conclusion, we do the research from the formal integration mechanism and socialization mechanism and focus on implicit knowledge and cultural differences. We find out that: 1) expatriates will transfer knowledge and skill to subsidiaries by company mechanism, 2) expatriates try to help subsidiaries to acquire advanced knowledge by their own relationship, 3) expatriates try to build a good relationship with other staff by cooperating with them, 4) expatriates will build a friendship with staff during their spare time. We put forward the following hypothesis:

H1-3: While expatriates are more actively seeking opportunities to transfer knowledge, the performance of subsidiary is better.

Cohen and Levinthal first identified the absorptive capacity of corporation, which means the ability of corporation to identify, understand and apply advantage knowledge. O Dell and Grayson found out whether employees as recipients have experience to share knowledge is very important to transfer knowledge as knowledge transfer is 
based on corporation members, which Qiang Xu identified as the ability to absorb and main knowledge [10]. The relationship to transfer inside the corporation plays an important role in the knowledge transfer among MNCs. Adam Smale found out that the basic absorptive capacities of corporations is to absorb practices and procedures, while it is very hard to absorb institutions and value. Lazarova and Tarque found out that the keys to affect the success to transfer knowledge are the willing of expatriates to transfer and the absorptive ability of organization. Nelson and Winter (1982) point out that corporations develops unique business practices, which aims to discover the function of its own technology and pass it to new users, and these will finally form the differences between corporations. X Martin \& R Salomon divide the ability to transfer knowledge into the ability of senders to transfer and the ability of recipient to absorb, and found out that contrast the abilities of both sides can do good to explain the conditions to select organizational structure. Lingfeng Yi came up with a model of expatriates to transfer knowledge.

We do the study from the perspective of expatriates, and focus on recipient absorptive capacity (RAC) [11]. Cohen and Levinthal identified RAC as that companies use existing knowledge to recognize the value of new knowledge, absorb and make full use of the new knowledge. EL-Sayed compared the process of knowledge transfer in MNCs and influence factors, and came up with an idea that it is the same important for MNCs that the motivation of MNCs to achieve knowledge, the absorptive capacity and the characteristics of knowledge, and even the motivation is more important. Foss and Pedersen put forward that the absorptive capacity of subsidiaries is stronger, the greater the likelihood of success of knowledge transfer happens. However, with the technology skill of subsidiaries becomes stronger, it can't avoid that subsidiaries have more power to judge independently and bargain with parent companies. Comprehensive previous conclusion, we measure the absorptive capacity of subsidiaries from six aspects: 1 ) whether subsidiaries have sufficient capacity to acquire the knowledge and skills from parent companies, 2) whether subsidiaries clearly understand what knowledge and skills to be learned, 3) whether subsidiary have sufficient accumulated technology and foundation to absorb the knowledge and skills from the parent company, 4) whether subsidiaries have sufficient capacity to implement knowledge and skills learning from parent companies, 5) whether subsidiaries can transform knowledge and skills learning from parent companies into their own knowledge, 6) whether subsidiaries have abilities to apply the latest research achievement of parent company to their own products. At the same time, based on the previous studies of knowledge transfer, the absorptive capacity of subsidiaries doesn't have a direct impact on performance. So in our study, we use the absorptive capacity of subsidiaries as a manipulated variable, then we put forward the following hypothesis:

H2-1: While the absorptive capacity of subsidiaries is stronger, knowledge transfer ability of expatriates plays a more important role to enhancing the performance of the subsidiary.

H2-2: While the absorptive capacity of subsidiaries is stronger, the motivation of expatriates plays a more important role to enhancing the performance of the subsidiary.

H2-3: While the absorptive capacity of subsidiaries is stronger, seeking opportunities to transfer knowledge plays a more important role to enhancing the performance of the subsidiary

\section{The Performance of Subsidiaries}

In the study, we measure the performance of subsidiaries of MNCs from operating performance, profit margin and rate of return on investment. As the effect of knowledge transfer needs time to accumulate, we should use dates of three to five years to measure the performance of subsidiaries.

\section{The Framework for Research}

Based on the analysis of precious researches, we put forward the researching framework among knowledge transfer of expatriates, the absorption capacity of subsidiaries and the performance of subsidiaries. As we can see in Figure 1, the competitive capacity of expatriates is positively correlated with the performance of subsidiaries, among which the absorptive capacity of subsidiaries is the mediator. In our study, we analyze the competitive capacity of expatriates from the ability to transfer knowledge, the motivation to transfer and seeking opportunity to transfer. From the precious research, we put forward four hypothesis, which are 1) the ability to transfer knowledge is positively correlated with the performance of subsidiaries; 2) the motivation to transfer is positively correlated with the performance of subsidiaries; 3 ) while expatriates are more actively seeking opportunities to transfer knowledge, the performance of subsidiary is better; 4) the absorptive capacity of subsidiaries as a mediator is positively correlated with the competitive capacity of expatriates, which is positively correlated with the performance of subsidiaries. If all these hypothesis are true, parent company should put the capacity of expatriates to transfer knowledge as the consideration criteria to decide send who to subsidiaries. For subsidiaries, the framework can help them to solve three problem, which are how to get better with expatriates, how to use their own decision-making power reasonable and how to 


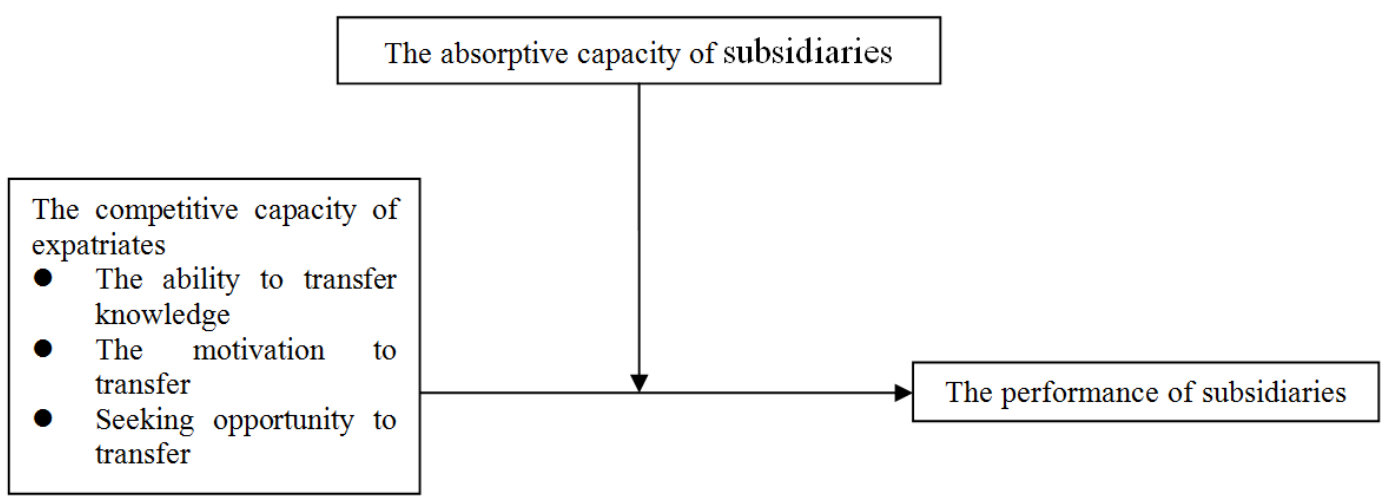

Figure 1. The researching framework among knowledge transfer of expatriates, the absorption capacity of subsidiaries and the performance of subsidiaries.

build a learning organization as soon as possible.

\section{Conclusion}

In the trend of globalization, the parent company of MNCs effectively transferring knowledge to subsidiaries can build a competitive advantage in subsidiaries. It is the common problem that MNCs will face how to make subsidiaries identify with the technology, institution and value of parent company, especially in alliances and acquisitions. In the study, we do the research from the aspect of expatriates, theoretically indentify three dimensions during the knowledge transfer among expatriates: ability, motivation and seeking for the opportunities to transfer knowledge, which is different from precious study only researching on the characteristics of knowledge and the number of expatriates. At the same time, we use adjustment variables of absorptive capacity into study, to measure the effect of situational factors. What's more, we use the life from establishment and the scale of subsidiaries as control variables, as the time to transfer and score to transfer will impact the effect of knowledge transfer. We also put the industry of subsidiary and the number of expatriates as control variables. Then we find the relationship among knowledge transfer of expatriates, the absorption capacity and the performance of subsidiaries. If all these hypothesis are true, parent company should put the capacity of expatriates to transfer knowledge as the consideration criteria to decide sending whom to subsidiaries. For parent company, in the process of mergers and alliances, managers should consider the absorptive capacity of subsidiary, in the order to create stable profit as soon as possible. For subsidiaries, the framework can help them solve three problems, which are how to get better with expatriates, how to use their own decision-making power reasonably and how to build a learning organization as soon as possible.

\section{REFERENCES}

[1] A. Edstrom and J. R. Galbraith, "Transfer of Managers as a Coordination and Control Strategy in Multinational Organizations,” Administrative Science Quarterly, Vol. 22, 1977.

[2] R. D. Arvey, W. Srtickland, G. Drauden and C. Martin, "Motivational Components of Test Taking," Personnel Psychology, Vol. 43, 1990, pp. 695-716.

[3] Y.-Y. Chang, Y. P. Gong and M. W. Peng, "Expatriate Knowledge Transfer, Subsidiary Absorptive Capacity and Subsidiary Performance," Academy of Management Journal.

[4] A. B. Antal, "Types of Knowledge Gained by Expatriate Managers,” Journal of General Management, Vol. 26, 2000.

[5] T. C. Chini, "Effective Knowledge Transfer in Multinational Corporations,” Macmillan, New York, 2004.

[6] E. L. Deci and R. Flaste, "Why We Do What We Do: The Dynamics of Personal Autonomy,” Putnam, New York, 1995.

[7] A. Perez-Araos and K. D. Barber, "Designing a Knowledge Management Tool to Support Knowledge Sharing Networks,” Journal of Manufacturing Technology Management, Vol. 18, 2007.

[8] A. C. Inkpen, "Managing Knowledge Transfer in International Alliances,” Thunderbird International Business Review, Vol. 50, 2008.

[9] J. M. Millar and J. Choic, "Reverse Knowledge and Technology Transfer: Imbalances Caused by Cognitive Barriers in Asymmetric Relationship," International Journal of Technology Management, Vol. 48, 2009, pp. 389-402.

[10] A. Smale, "Global HRM Integration: A Knowledge Transfer Perspective,” Personnel Review, Vol. 37, No. 2, 2008.

[11] El-Sayed, “An Ontology-Based Approach to Inter-Organizational Knowledge Transfer," Journal of Global Information Technology Management. 